\title{
Hiding and finding: The relationship between visual concealment and visual search
}

\author{
Daniel Smilek, Laura Weinheimer, and Donna Kwan \\ University of Waterloo, Waterloo, Ontario, Canada \\ Mike ReYnolds \\ Trent University, Peterborough, Ontario, Canada \\ AND \\ Alan Kingstone \\ University of British Columbia, Vancouver, British Columbia, Canada
}

\begin{abstract}
As an initial step toward developing a theory of visual concealment, we assessed whether people would use factors known to influence visual search difficulty when the degree of concealment of objects among distractors was varied. In Experiment 1, participants arranged search objects (shapes, emotional faces, and graphemes) to create displays in which the targets were in plain sight but were either easy or hard to find. Analyses of easy and hard displays created during Experiment 1 revealed that the participants reliably used factors known to influence search difficulty (e.g., eccentricity, target-distractor similarity, presence/absence of a feature) to vary the difficulty of search across displays. In Experiment 2, a new participant group searched for the targets in the displays created by the participants in Experiment 1. Results indicated that search was more difficult in the hard than in the easy condition. In Experiments 3 and 4, participants used presence versus absence of a feature to vary search difficulty with several novel stimulus sets. Taken together, the results reveal a close link between the factors that govern concealment and the factors known to influence search difficulty, suggesting that a visual search theory can be extended to form the basis of a theory of visual concealment.
\end{abstract}

Since $9 / 11$, there has been a growing focus on increasing security in public places such as airports. Laboratory research on this security issue has tended to capitalize on a large body of work examining how people visually search for a target object embedded among a set of distractor objects (e.g., Bundesen, 1990; Duncan \& Humphreys, 1989; Treisman \& Gelade, 1980; Wolfe, 1994; Wolfe, Cave, \& Franzel, 1989). In these studies, participants are typically required to search for a target that is placed in plain sight among a group of distractor items. Table 1 presents a summary of some of the factors that have been shown to influence search, together with a description of the influence.

Naturally, the act of visual search often requires searching for something that is hidden. Despite this close interrelationship between hiding and search, research has focused almost entirely on the act of search. Here, we explore the related act of hiding and take some initial steps toward developing a psychological theory of concealment. Our specific hypothesis was that because hiding and search are logically closely coupled, the basic principles that govern how people hide objects will be closely related to the basic principles that govern visual search.
We began by evaluating whether naive participants would use the same factors that have been shown to govern search difficulty when they were required to arrange objects in a manner that they believed would make a target either easy or hard to find. To closely match the visual search literature, which involves finding objects in plain sight, we focused on the concealment of objects in plain sight (i.e., without occlusion). In Experiment 1, participants were asked to complete a placement task in which they had to create visual search displays such that a target would be either easy or hard to find. In this task, the participants were given three stimulus sets (see Figure 1) and were asked to place the objects on a display surface such that the target was either easy or hard to find. Each of the stimulus sets was chosen because it had previously been employed in studies of search. The first stimulus set, the asymmetry-shape set, was composed of a circle and a circle with a line through it and has been used in previous studies of visual search that evaluated how the presence or absence of a single visual feature influences search difficulty (see Treisman \& Gormican, 1988; Treisman \& Souther, 1985). The second stimulus set, the affective-face set, which included three different affective faces, has been 
Table 1

Eight Factors Known to Influence Search Difficulty, Together With a Brief Description of Their Influence on Search and Corresponding References

\begin{tabular}{|c|c|c|}
\hline Factor & Influence on Visual Search & References \\
\hline Target eccentricity & $\begin{array}{l}\text { Search efficiency decreases as target eccentricity } \\
\text { increases. }\end{array}$ & $\begin{array}{l}\text { Carrasco, Evert, Chang, \& Katz, 1995; Carrasco \& Frieder, } \\
\text { 1997; Carrasco, McLean, Katz, \& Frieder, 1998; Carrasco, } \\
\text { Talgar, \& Cameron, 2001; Carrasco \& Yeshurun, } 1998\end{array}$ \\
\hline Organization & $\begin{array}{l}\text { Search is more efficient when distractors are organized } \\
\text { in such a way that they individuate or direct attention to } \\
\text { the target. }\end{array}$ & $\begin{array}{l}\text { Hooge \& Erkelens, 1998; Moraglia, 1989; Olds, Cowan, } \\
\text { \& Jolicœur, 1999; Wolfe, Friedman-Hill, Stewart, \& } \\
\text { O'Connell, } 1992\end{array}$ \\
\hline $\begin{array}{l}\text { Orientational } \\
\text { heterogeneity }\end{array}$ & $\begin{array}{l}\text { Search efficiency decreases as the orientations of the } \\
\text { items in the display become more heterogeneous. }\end{array}$ & $\begin{array}{l}\text { Duncan \& Humphreys, 1989; Moraglia, 1989; } \\
\text { Wolfe et al., } 1992\end{array}$ \\
\hline $\begin{array}{l}\text { Target-distractor } \\
\quad \text { similarity }\end{array}$ & $\begin{array}{l}\text { Search efficiency increases as the similarity between } \\
\text { target and distractors decreases. }\end{array}$ & Duncan \& Humphreys, 1989 \\
\hline $\begin{array}{l}\text { Presence or absence } \\
\text { of a feature }\end{array}$ & $\begin{array}{l}\text { Search is more efficient when the target contains a feature } \\
\text { not present in the distractors than when it is defined by } \\
\text { the absence of a feature present in the distractors. }\end{array}$ & Treisman \& Gormican, 1988; Treisman \& Souther, 1985 \\
\hline Emotional valence & $\begin{array}{l}\text { Search for negative faces is more efficient than search for } \\
\text { positive faces. }\end{array}$ & $\begin{array}{l}\text { Eastwood, Smilek, \& Merikle, 2001; Fox et al., 2000; } \\
\text { Hansen \& Hansen, 1988; Ohman, Lundqvist, \& Esteves, } \\
2001\end{array}$ \\
\hline $\begin{array}{l}\text { Categorical } \\
\text { membership }\end{array}$ & $\begin{array}{l}\text { Search is more efficient when the target and distractors } \\
\text { belong to different semantic categories than when the } \\
\text { target and distractors belong to the same semantic } \\
\text { category. }\end{array}$ & $\begin{array}{l}\text { Brand, 1971; Gleitman \& Jonides, 1978; Ingling, 1972; } \\
\text { Jonides \& Gleitman, 1972, } 1976\end{array}$ \\
\hline
\end{tabular}

used in studies of emotional valence in visual search (see Eastwood, Smilek, \& Merikle, 2001; Fox et al., 2000). Finally, the third stimulus set, the alphanumeric set, was composed of letters and numbers. Alphanumeric stimuli are commonly used in studies of visual search and have been used to evaluate the role of category membership on attentional deployment (see Brand, 1971; Ingling, 1972; Jonides \& Gleitman, 1972).

The easy and hard search displays created by the participants during the placement task were coded on up to seven factors that have previously been examined in laboratory studies of visual search. The seven factors are shown in Table 1 and include (1) target eccentricity, (2) spatial organization of search items, (3) orientational heterogeneity of the search items, (4) target-distractor similarity, (5) the presence versus absence of a feature defining the target (search asymmetries), (6) the emotional valence of the search items, and (7) the categorical membership of the search items. There were several reasons for choosing this subset of all possible factors. First, we chose target eccentricity, organization, orientational heterogeneity, target-distractor similarity, and presence versus absence of a feature because these have been some of the most well-established factors in the literature on visual search (e.g., Carrasco \& Frieder, 1997; Duncan \& Humphreys, 1989; Treisman \& Gormican, 1988; see Wolfe, 1998, for a review). Second, we chose to evaluate emotional valence and category membership because they have received much attention in studies of search but their effects on search continue to be debated (see Duncan, 1983; Frischen, Eastwood, \& Smilek, 2008; Purcell, Stewart, \& Skov, 1996; Smilek, Dixon, \& Merikle, 2006).

We expected that Experiment 1 would reveal that the same factors that have been shown to govern search difficulty also influence how people place objects so that they are either easy or hard to find. If our expectation is correct, it seems reasonable to suggest that visual search theory can be extended to form a foundation for a theory of the visual aspects of concealment.

\section{EXPERIMENT 1}

\section{Method}

Participants. Two groups of students from the University of Waterloo participated in a 1-h experimental session. One group (the paid group) consisted of 32 students who participated in exchange for $\$ 8$. The second group (the credit group) consisted of 24 students who participated in exchange for a partial course credit in their introductory psychology class. We tested introductory psychology students because it was highly unlikely that they had received any formal instruction about the factors that govern search difficulty. Because the results did not differ across groups in any important way, the data from the two groups were combined for analysis. All the participants reported normal or corrected-to-normal vision.

Materials. Figure 1 shows the three distinct stimulus sets used in the placement task. The first set included a circle and a circle with a

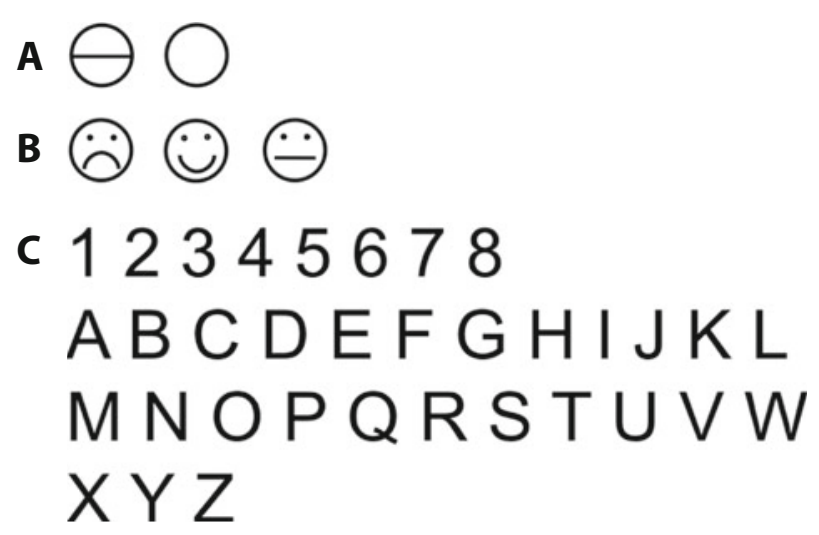

Figure 1. Stimulus sets used for the placement task in Experiment 1. (A) Asymmetry-shape set. (B) Affective-face set. (C) Alphanumeric set. 
A

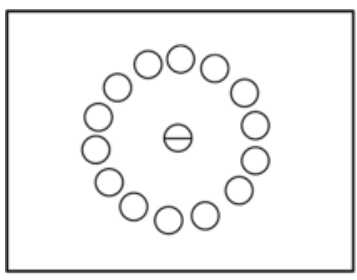

C

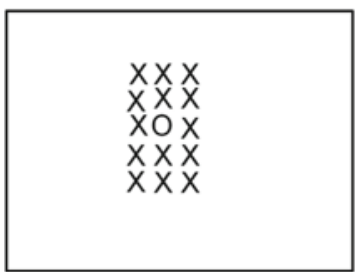

B

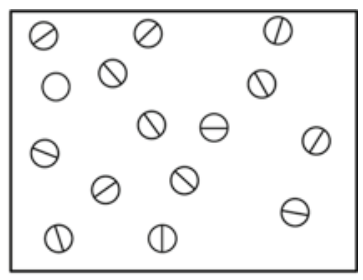

D

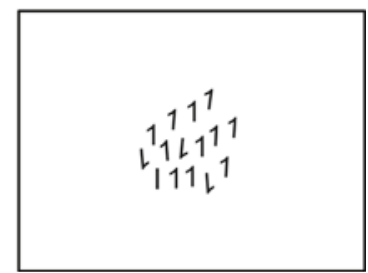

Figure 2. Examples of easy and hard search displays created by participants. (A and B) Easy and hard displays, respectively, created by a single participant using the asymmetry-shape set. $A$ comparison of panels $A$ and $B$ shows that the participant used search asymmetry, organization, and eccentricity to vary search difficulty. (C and D) Easy and hard displays, respectively, created by a different participant using the alphanumeric set. $A$ comparison of panels $C$ and $D$ shows that the participant used target-distractor similarity and orientational heterogeneity to vary search difficulty.

line through it (the asymmetry-shape set). The second set included three schematic faces expressing a positive, negative, or neutral emotion (the affective-face set). The third stimulus set included all uppercase letters and the numbers 1 through 8 (the alphanumeric set). Excluded from this set were the numbers " 0 " and "9," because they were visually identical to the letter "O" and the number " 6 ," respectively. Each of the stimuli used in the first two sets was printed on a square piece of paper measuring $5 \times 5 \mathrm{~cm}$. The stimuli used in the alphanumeric set were $2.5 \mathrm{~cm}$ in width and $3.75 \mathrm{~cm}$ in height and were made from a thin wooden board. There were 14 instances of each of the stimuli. The participants placed the stimuli on a horizontal surface measuring $40.6 \mathrm{~cm}$ in width $\times 51 \mathrm{~cm}$ in height. The area on which the participants were to place the search stimuli was outlined on the surface of a desk by a border made of masking tape.

Procedure. As a warm-up, the participants first completed a drawing task in which they were given a sheet of paper and instructed to draw a target object (either a circle or a cross) on the page and then to add any number of pencil marks on the page to make the target either easy or hard to find. The results of this task will not be discussed further.

The participants were then required to make easy and hard search displays, using the three stimulus sets shown in Figure 1. At the beginning of a trial, 14 instances of each object in a given stimulus set were placed in front of the participants, with separate, neat piles for each type of object. The participants were specifically instructed to choose one of the objects to be the target and 14 instances of another object in the set to be the distractors. Thus, for each display, there was one unique target object and 14 instances of the distractor object. The participants were instructed to place the target and distractors within the outlined space in whatever arrangement they wanted, as long as the objects did not overlap. Critically, the participants were instructed to place the objects so that the target was either easy or hard to find. Thus, for each of the three stimulus sets, the participants created two displays: one in which it would be hard to find the target (hard condition) and another where it was easy to find the target (easy condition). All together, each participant created a total of six displays.

Each display was photographically recorded using a Nikon Coolpix 2200 digital camera. The participants always created the hard and easy displays for the asymmetry-shape set first, then for the affective-faces set, and finally for the alphanumeric set. The order of the easy and hard conditions was counterbalanced across participants.

\section{Results and Discussion}

In order to control variability due to picture quality (lighting, shadows, pixilation, etc.), the search displays created by the participants were re-created using PowerPoint (Microsoft, 2003) prior to coding the displays. Examples of two easy and two hard displays are shown in Figure 2. The displays were then coded on the eight factors described in the introduction by two independent coders. The specific coding scheme for each factor and the corresponding results will be discussed for each factor in turn.

Eccentricity. Target eccentricity was defined as the distance of the target item from the center of the display. Eccentricity was coded by placing a bull's-eye grid on each display. The bull's-eye had seven levels of eccentricity, which corresponded to the 7 points on a Likert scale, with targets close to the center being scored as a " 1 " and those in the most eccentric circle of the bull's-eye being scored as a "7." The easy and hard search displays for each stimulus set were coded separately by two independent coders. The interrater reliability of the coders was very high $(r=.98)$, and so the scores were averaged across coders. The resulting scores were submitted to a 2 (difficulty: easy vs. hard) $\times 3$ (stimulus set: asymmetry-shape vs. affective-face vs. alphanumeric) repeated measures ANOVA. The mean scores for each condition are shown in Table 2. As can be seen in the table, targets were placed closer to the center of the display in the easy condition than in the hard condition $\left[F(1,155)=22.3, M S_{\mathrm{e}}=1.90\right.$, $p<.001]$. The overall eccentricity of the targets also dif-

Table 2

Mean Scores on the Eccentricity, Organization, and Orientational Heterogeneity Dimensions As a Function of Stimulus Set and Search Difficulty in Experiment 1

\begin{tabular}{lcccccc}
\hline \multicolumn{1}{c}{ Dimension } & \multicolumn{2}{c}{ Difficulty } & & & \\
\cline { 2 - 3 } & Easy & Hard & Difference & $t$ & $p$ \\
\hline Eccentricity & & & & & & \\
$\quad$ Asymmetry-shape & 2.9 & 3.9 & 1.0 & 4.6 & .00 \\
Emotional-valence & 3.0 & 3.6 & 0.7 & 3.3 & .00 \\
Alphanumeric & 2.7 & 3.1 & 0.5 & 2.4 & .02 \\
Organization & & & & & \\
Asymmetry-shape & 6.4 & 3.9 & -2.5 & 10.4 & .00 \\
Emotional-valence & 6.0 & 4.2 & -1.8 & 6.9 & .00 \\
Alphanumeric & 6.0 & 4.6 & -1.4 & 5.0 & .00 \\
Orientational heterogeneity & & & & & \\
Asymmetry-shape & 1.8 & 4.2 & 2.4 & 8.0 & .00 \\
Emotional-valence & 1.6 & 4.2 & 2.6 & 9.0 & .00 \\
Alphanumeric & 2.8 & 4.1 & 1.4 & 3.9 & .00 \\
\hline
\end{tabular}

Note-Difference scores are calculated as scores in the hard condition minus the scores in the easy condition. All $t$ tests are repeated measures, two-tailed tests. 
fered between the stimulus sets $\left[F(2,110)=8.4, M S_{\mathrm{e}}=\right.$ $1.06, p<.001]$, with the most eccentric targets occurring in the asymmetry-shape set and the least eccentric targets occurring in the alphanumeric set. Individual repeated measures $t$ tests comparing the scores in the easy and hard conditions were conducted for each stimulus set and are shown in Table 2. Overall, these findings suggest that the participants used eccentricity, a factor known to influence visual search in previous behavioral studies (e.g., Carrasco, Evert, Chang, \& Katz, 1995), to vary the degree to which objects were concealed in plain sight.

Organization. The organization of the items in the easy and hard search displays for each stimulus set was also scored by two coders using a 7-point Likert scale. Organization was defined as the degree of structure and order of the stimuli in the display. A display was scored as a "1" when the stimuli lacked any clear geometrical organization or symmetry and were just randomly placed in the display. On the other extreme, a display was given a " 7 " when the stimuli in the display formed geometric shapes or simple patterns or were arranged in a symmetrical fashion. The scores were averaged across coders because the interrater reliability of this dimension was high $(r=.85)$. Table 2 shows the resulting mean scores for the easy and hard conditions associated with each stimulus set. A 2 (difficulty: easy vs. hard) $\times 3$ (stimulus set: asymmetry-shape vs. affective-face vs. alphanumeric) repeated measures ANOVA revealed that search displays in the easy condition had a greater degree of spatial organization and structure than did those in the difficult condition $\left[F(1,55)=100.2, M S_{\mathrm{e}}=2.94, p<.001\right]$ and that organization was used about equally across the three stimulus sets $\left[F(2,110)=1.14, M S_{\mathrm{e}}=1.14, p=\right.$ .32]. There was also an interaction between difficulty and stimulus set $\left[F(2,110)=6.1, M S_{\mathrm{e}}=1.34, p<.01\right]$, with the largest effect of difficulty in the asymmetry-shape set and the smallest effect in the alphanumeric set. Repeated measures $t$ tests revealed that the effect of difficulty was significant for each stimulus set (see Table 2). Thus, the participants made use of spatial organization consistently across the three stimulus sets to vary the degree of concealment of objects. As with the previous dimension, the participants' intuitions about how organization influences search seem to match previous findings in the search literature (see Hooge \& Erkelens, 1998; Moraglia, 1989; Olds, Cowan, \& Jolicœur, 1999).

Orientational heterogeneity. The heterogeneity in orientation (i.e., tilt) of the items in the display was coded by two coders using a 7-point Likert scale, with a "1" given when the items were oriented in the same direction and a "7" given when the items were oriented in different (e.g., random) directions. The interrater reliability was again very high $(r=.90)$, and the scores were averaged across coders. The data were submitted to a 2 (difficulty: easy vs. hard) $\times 3$ (stimulus set: asymmetry-shape vs. affectiveface vs. alphanumeric) repeated measures ANOVA. The mean scores for the easy and hard displays corresponding to each stimulus set are shown in Table 2. Critically, stimulus orientation was more heterogeneous in the hard condition than in the easy condition $\left[F(1,55)=106.2, M S_{\mathrm{e}}=\right.$
$3.59, p<.001]$. The interaction between difficulty and stimulus set was also significant $\left[F(2,110)=5.2, M S_{\mathrm{e}}=\right.$ $2.38, p<.01]$, with the alphanumeric set exhibiting a smaller effect of difficulty than did the other stimulus sets. There was also a main effect of stimulus set $[F(2,110)=$ $\left.5.6, M S_{\mathrm{e}}=1.74, p<.01\right]$, with greater heterogeneity in stimulus orientation in the alphanumeric set than in the asymmetry-shape and affective-face sets. The greater orientational homogeneity in the alphanumeric stimulus set appears to be due to people's using stimulus orientation both to help direct attention toward the target in the easy condition (the target "O" in the center of a circle of "I" distractors, each pointing to the target) and to increase featural overlap (e.g., rotating a "W" distractor $180^{\circ}$ to match an " $\mathrm{M}$ " target). Repeated measures $t$ tests showed strong effects of difficulty for each stimulus set (see Table 2). These findings indicate that participants use the orientation of items in the display when varying the degree of concealment of an object, which matches nicely with behavioral findings using the standard visual search paradigm (e.g., Duncan \& Humphreys, 1989; Moraglia, 1989; Wolfe, Friedman-Hill, Stewart, \& O'Connell, 1992).

Target-distractor similarity. The use of targetdistractor similarity was evaluated using the alphanumeric stimulus set, because this set involved the greatest variety of targets and distractors. Target-distractor similarity was measured by comparing featural overlap. At present, there is no definitive similarity set of letter features; therefore, two different feature sets were used. One set (Gibson, Osser, Schiff, \& Smith, 1963) consisted of qualitatively different non-position-specific features (e.g., a straight line [horizontal, vertical, or diagonal], curved line, symmetry). The other feature set (Rumelhart \& Siple, 1974) consisted of position-specific features. The latter feature set has been used in a number of computational models (e.g., Coltheart, Rastle, Perry, Langdon, \& Ziegler, 2001; Rumelhart \& McClelland, 1982). For each target-distractor combination, we measured how many of the total possible features were shared between the target and distractor items. We included both shared presence and shared absence in the total. This resulted in a possible range of scores from 0 (very dissimilar) to 12 (identical) for the Gibson et al. feature set and 0 (very dissimilar) to 14 (identical) for the Rumelhart and Siple feature set. The resulting similarity scores were submitted to a paired-sample $t$ test to evaluate whether there was a difference in the use of target-distractor similarity for easy and hard search displays. The analysis revealed that target-distractor similarity was higher in the hard than in the easy condition, using both the Gibson et al. features [6.7 and 4.0, respectively; $t(55)=4.22, p<.001]$ and the Rumelhart and Siple features [10.9 and 6.7 features, respectively; $t(55)=8.7, p<.001] .{ }^{1}$ This result indicates that participants intuit, consistent with previous search findings (Duncan \& Humphreys, 1989), that targetdistractor similarity influences search difficulty.

Presence versus absence of a feature (search asymmetries). The asymmetry-shape stimulus set was used to assess whether participants can intuit that search is more efficient when targets are defined by the presence of a 
Table 3

Frequency With Which Each Target-Distractor Pair Was Chosen When Easy and Hard Search Displays Were Created Using the Affective-Face Set in Experiment 1

\begin{tabular}{|c|c|c|c|}
\hline \multicolumn{2}{|c|}{ Stimulus } & \multicolumn{2}{|c|}{ Difficulty } \\
\hline Target & Distractor & Easy & Hard \\
\hline (;) & : & 9 & 18 \\
\hline : & ;) & 6 & 29 \\
\hline (;) & 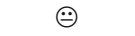 & 22 & 2 \\
\hline$\oplus$ & (;) & 11 & 3 \\
\hline : & $\oplus$ & 4 & 0 \\
\hline 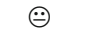 & : & 4 & 4 \\
\hline
\end{tabular}

feature than when targets are defined by the absence of a feature (i.e., search asymmetry). The asymmetry-shape set was useful for this analysis because it consisted of two circle stimuli, one of which had an extra line segment feature. To evaluate knowledge of search asymmetries, the participants were assigned a score of " 1 " if they chose the target with a feature present and " 0 " if they chose the target with the feature absent. The results clearly showed that the participants used the item with an extra feature present as the target on a larger proportion of the easy displays (.61) than of the hard displays (.21). A repeated measures $t$ test assessing the use of the stimulus with the extra feature in the easy and hard search displays revealed a significant effect of display difficulty $[t(1,55)=4.718$, $p<.001]$. We find this result striking, because it implies that people can intuit that subtle display asymmetries can influence the difficulty of visual search and use it when concealing objects to different degrees. ${ }^{2}$ Once again, the factor people use to vary concealment matches behavioral investigations of visual search asymmetries (see Treisman \& Gormican, 1988; Treisman \& Souther, 1985).

Emotional valence. The affective-face stimulus set was used to evaluate whether people intuit that emotional valence can influence search difficulty. The analysis consisted of counting the number of hard and easy displays in which each target-distractor combination was used. Since the faces in this set expressed three emotions (positive, neutral, and negative), there was a total of six possible target-distractor pairings. Table 3 shows the number of displays that contained each target-distractor pair. The participants were assigned a score of " 1 " for each of the target-distractor pairs that they used and a " 0 " for the ones that they did not use.

We first assessed whether the participants intuited the general finding that negative faces embedded among positive faces are found more efficiently than positive faces embedded among negative faces (e.g., Hansen \& Hansen, 1988). The data from the positive-negative and the negative-positive pairs were submitted to an ANOVA with stimulus pair (positive-negative vs. negativepositive) and search difficulty (easy vs. hard) as withinparticipants factors. The results revealed that these two stimulus pairs (positive-negative vs. negative-positive) were used much more in the hard displays than in the easy displays $\left[F(1,55)=39.1, M S_{\mathrm{e}}=0.117, p<.001\right]$, and there were no overall differences between the frequency of use of the two stimulus pairings ( $F<1.07, p>.30$ ). The interaction was marginally significant $[F(1,55)=3.182$, $\left.M S_{\mathrm{e}}=0.27, p=.08\right]$, reflecting that people tended to use negative targets embedded among positive distractors more often than the reverse to make search targets hard to find, which is actually opposite to what is typically found in the literature. Next, we assessed whether the participants intuited the finding that negative faces embedded among neutral distractors are found more efficiently than positive faces embedded among neutral distractors (see Eastwood et al., 2001). An ANOVA assessing stimulus pair (positive-neutral vs. negative-neutral) and search difficulty (easy vs. hard) as within-participants factors revealed a significant effect of difficulty $[F(1,55)=38.5$, $\left.M S_{\mathrm{e}}=0.091, p<.001\right]$, a marginally significant effect of stimulus pair $\left[F(1,55)=3.0, M S_{\mathrm{e}}=0.146, p=.09\right]$, and again a marginally significant interaction $[F(1,55)=3.6$, $\left.M S_{\mathrm{e}}=0.179, p=.06\right]$. Interestingly, however, in contrast to the findings in the literature, if anything, the participants intuited that positive faces among neutral distractors would be easier to find than negative faces among neutral distractors. These findings are suggestive of the conclusion that the participants used facial affect to vary search difficulty but that they used it in a different way than has been reported in studies of visual search. This being said, it is important to highlight that the influence of facial emotion on attention appears to be very contextualized and subtle, likely accounting for the relatively low amount of variance in laboratory search studies (Gerritsen, Frischen, Blake, Smilek, \& Eastwood, 2008; Hunt, Cooper, Hungr, \& Kingstone, 2007; see Frischen et al., 2008, for a review).

Category membership. The specific target-distractor combinations used when the participants created displays with the alphanumeric stimulus set were analyzed to evaluate whether the participants intuited that category membership of targets and distractors influences search difficulty. Because the participants had a choice of using either numbers or letters to create search displays, there were four possible categorical combinations of target and distractor pairs. The four combinations are shown in Table 4, together with the number of easy and hard search displays that included each of these combinations of targetdistractor categories. Inspection of Table 4 clearly shows that, collectively, the participants did not make use of the category effect; indeed, most of the displays used a target letter embedded among letter distractors. To statistically analyze these data, the participants were assigned a score of " 1 " for each of the target-distractor pairs that they used

Table 4

Frequency With Which Each Target-Distractor Pair Was Chosen When Easy and Hard Search Displays Were Created Using the Alphanumeric Set in Experiment 1

\begin{tabular}{lllrr}
\hline \multicolumn{2}{c}{ Stimulus } & & \multicolumn{2}{c}{ Difficulty } \\
\cline { 2 - 2 } \cline { 5 - 5 } Target & Distractor & & Easy & Hard \\
\hline Letter & number & & 7 & 8 \\
Number & letter & & 6 & 11 \\
Letter & letter & & 41 & 36 \\
Number & number & 2 & 1 \\
\hline
\end{tabular}


and a " 0 " for the ones that they did not use. Each of the target-distractor pairs were then evaluated individually with a repeated measures $t$ test to assess the use of that target-distractor pair in the easy and hard search displays. The analyses showed that each of the target-distractor pairs was used roughly equally often in the easy and hard search displays (all $t \mathrm{~s}<1.299$, all $p \mathrm{~s}>.199$ ).

\section{Summary}

Overall, the results of Experiment 1 support the general conclusion that people use factors known from laboratory studies to influence search difficulty (e.g., eccentricity, organization, orientation, target-distractor similarity, search asymmetry) when concealing objects in plain sight to different degrees. We were particularly struck by the fact that the participants were sensitive to search asymmetries, because it is typically thought that search asymmetries are subtle constructs developed by researchers. The fact that the participants used search asymmetries to vary degree of concealment implies that they had access to very subtle factors that govern search difficulty. In addition, although the results were suggestive of the conclusion that the participants used emotional valence to vary search difficulty, they did this in a way that was different from what has typically been shown in the search literature. Finally, the participants did not use the category effect to vary search difficulty. However, since we did not ask the participants to optimize search difficulty or ease, it may be that they did have intuitive knowledge about the category effect but simply chose to use other factors (e.g., orientational heterogeneity and eccentricity) to vary search difficulty.

\section{EXPERIMENT 2}

In Experiment 2, we sought to confirm that the participants in Experiment 1 were, in fact, successful at creating easy and hard search displays. To this end, all of the displays from the placement task in Experiment 1 were compiled and used as displays in a standard visual search task. If the participants in Experiment 1 were successful at creating easy and hard search displays, we would expect that search performance should be faster and more accurate for easy than for hard search displays.

\section{Method}

Participants. Twenty undergraduate students from the University of Waterloo participated in a half-hour session in exchange for course credit in their introductory psychology class. Ten participants searched displays created by the 32 participants in the paid group in Experiment 1, and the other 10 participants searched displays created by the 24 participants in the credit group in Experiment 1. All the participants reported normal or corrected-to-normal vision.

Materials. In order to control variability due to picture quality (lighting, shadows, pixilation, etc.), the search displays created by the participants in the placement task in Experiment 1 were re-created using Microsoft PowerPoint 2003. The PowerPoint versions of the search displays served as search displays in the present experiment. For participants searching displays created by the 32 participants in the paid group in Experiment 1, there was a total of 192 displays, of which 64 included the asymmetry-shape set, 64 included the affectiveface set, and 64 included the alphanumeric set. For the participants searching displays created by the 24 participants in the course credit group in Experiment 1, there was a total of 144 displays, of which 48 included the asymmetry-shape set, 48 included the affective-face set, and 48 included the alphanumeric set. Half of the displays were created to be easy, and half were created to be hard. Each display contained 15 search objects (one target and 14 distractors) that measured approximately $1.5 \mathrm{~cm}$ vertically and $1.0 \mathrm{~cm}$ horizontally. At a viewing distance of $57 \mathrm{~cm}$, the stimuli measured $1.5^{\circ}$ vertically and $1.0^{\circ}$ horizontally. The search objects were presented in black against a white background. The displays were presented on a ViewSonic G70f monitor, and the presentation of the displays was controlled by E-Prime experimental software, Version 1.1 (Schneider, Eschman, $\&$ Zuccolotto, 2001), running on a computer with a 3.00-GHz Pentium 4 processor.

Procedure. On each trial in the experiment, the participants were presented with a visual search display and were required to search for the unique target in the display as quickly as possible without sacrificing accuracy for speed. Participant response time (RT) and accuracy were recorded. Each trial began with a fixation cross at the center of the screen. Once the fixation cross was presented, the participants depressed the " $n$ " button on the keyboard to trigger the onset of the visual search display. The participants held down the " $n$ " button on the keyboard while searching for the target and released the button as soon as they found the target. In this way, it was possible to measure the RT associated with the search. When the participants released the button, the display disappeared from the screen. The participants were then asked to point to the location where the target had been in the preceding search display. The screen remained blank until the research assistant indicated participant accuracy. The research assistant coded the accuracy with which the participants correctly identified the target by keying in " 1 " when the participants correctly pointed to the target and " 0 " when the participants did not correctly point to the target. The research assistant's coding of accuracy triggered the next trial of the experiment.

\section{Results and Discussion}

Response times. The data from all 20 participants were combined in a single analysis. Before analyzing the RT data, all RTs associated with error responses were removed. The data were then subjected to outlier trimming; RTs that were more than three standard deviations away from the mean in their respective cells were recursively excluded from analyses. Outlier trimming resulted in the removal of $4.6 \%$ of correct RTs. The mean RTs for the hard and easy conditions of each stimulus set were then calculated for each participant. The resulting data were analyzed using a 2 (difficulty: hard vs. easy) $\times 3$ (stimulus set: asymmetry-shape set vs. affective-face set vs. alphanumeric set) repeated measures ANOVA.

The mean RTs for each condition, averaged across participants, are shown in Table 5. As can be seen in Table 5, overall, RTs differed for the three stimulus sets $[F(2,38)=$ 92.6, $\left.M S_{\mathrm{e}}=67,793, p<.001\right]$, with search being most difficult with affective-face stimuli, moderately difficult with alphanumeric stimuli, and least difficult with asymmetryshape stimuli. More important, the table also shows that search was substantially less efficient for displays created to be hard searches than for displays created to be easy searches $\left[F(1,19)=85.5, M S_{\mathrm{e}}=156,847, p<.001\right]$. There was also a significant interaction between difficulty and stimulus set $\left[F(2,38)=50.5, M S_{\mathrm{e}}=37,796, p<.001\right]$, with greater effects of difficulty in the affective-face and alphanumeric sets than in the asymmetry-shape set. Separate repeated measures $t$ tests evaluating difficulty (easy vs. hard) revealed significant effects of difficulty for each set 
Table 5

Mean Response Times (in Milliseconds) and Errors (As Percentages) for Localizing the Target in the Easy and Hard Displays for Each Stimulus Set in Experiment 2

\begin{tabular}{lrrrrrr}
\hline & \multicolumn{2}{c}{ Difficulty } & & & \\
\cline { 2 - 4 } & Easy & Hard & Difference & $t$ & $p$ \\
\hline Reaction time & & & & & & \\
$\quad$ Asymmetry-shape & 681 & 902 & 221 & 6.0 & $<.01$ \\
Emotional-valence & 1,018 & 2,112 & 806 & 8.9 & $<.01$ \\
Alphanumeric & 682 & 1,373 & 691 & 8.4 & $<.01$ \\
Percent error & & & & & \\
Asymmetry-shape & 0.4 & 1.2 & 0.8 & 1.6 & .14 \\
Emotional-valence & 0.8 & 4.3 & 3.5 & 2.4 & .03 \\
Alphanumeric & 0.3 & 3.6 & 3.4 & 3.4 & $<.01$ \\
\hline
\end{tabular}

Note-Difference scores were calculated by subtracting scores in the easy from scores in the hard difficulty conditions. All $t$ tests were twotailed.

(see Table 5). These results clearly show that the participants in Experiment 1 had excellent intuitions about search and were successful at creating easy and hard search displays using factors such as eccentricity, organization, orientation, target-distractor similarity, and search asymmetries. Moreover, even though the participants did not make strong use of emotional valence or category membership to vary search difficulty of displays with the affective-face and the alphanumeric stimuli, respectively, they were nevertheless able to successfully vary difficulty using other factors.

Errors. The percentages of errors in each condition are also shown in Table 5. As can be seen in the table, there was a marginal difference in percentage of error across the three stimulus sets $\left[F(2,38)=2.7, M S_{\mathrm{e}}=10.97, p=.08\right]$, with error being greatest for the alphanumeric set, moderate for the affective-face set, and least for the asymmetryshape set. The table also shows that there were substantially more errors for displays created to be hard searches than for displays created to be easy searches $[F(1,19)=$ $\left.16.3, M S_{\mathrm{e}}=11.64, p<.01\right]$. There was also a marginal interaction between difficulty and stimulus set $[F(2,38)=$ $\left.2.8, M S_{\mathrm{e}}=8.32, p=.07\right]$, with greater effects of difficulty in the alphanumeric set and affective-face set than in the asymmetry-shape set. Separate repeated measures $t$ tests evaluating difficulty (easy vs. hard) for each stimulus set revealed significant effects of difficulty in each set (see Table 5). These results provide additional evidence that the participants in Experiment 1 had excellent intuitions about search and were successful at creating easy and hard search displays.

\section{EXPERIMENT 3}

The purpose of Experiment 3 was threefold. The first goal was to explore the generality of our finding that people can intuit search asymmetries (Experiment 1). The finding that people can intuit search asymmetries is particularly interesting because search asymmetries are typically thought to be quite a subtle factor that would normally not be easily intuited. Recall that in Experiment 1, we included only one type of search asymmetry stimulus pair (circle vs. circle with a line). In Experiment 3, we included three different search asymmetry pairs: long line versus short line, double line versus single line, and curved line versus straight line. Each of these stimulus sets is shown in Figure 3. We included these pairs because they have each been shown to yield substantial search asymmetries in studies of visual search (Treisman \& Gormican, 1988). The second goal was to evaluate in a more objective manner whether participants use target eccentricity and orientational heterogeneity to influence the difficulty of search. These two dimensions were scored subjectively by raters and objectively by direct measurement to evaluate whether the subjective ratings matched the objective measures. Finally, we sought to evaluate whether target eccentricity and orientational heterogeneity are varied in a correlated manner or whether they are varied independently of each other. The participants were given the three search asymmetry sets and, as in Experiment 1, completed a placement task in which they created search displays such that the targets were either easy or hard to find.

\section{Method}

Participants. Twelve undergraduate students at the University of Waterloo participated in a 1-h session in exchange for partial course credit in their introductory psychology class. All the participants reported having normal or corrected-to-normal vision.

Materials. The materials were similar to those used for the placement task in Experiment 1, with the exception that different stimulus sets were used in the present experiment. Figure 4 shows the three search asymmetry stimulus pairs used in the present experiment: long line (feature present) versus short line (feature absent), double line (feature present) versus single line (feature absent), and curved line (feature present) versus straight line (feature absent).

Procedure. The procedure was similar to that in Experiment 1 As in Experiment 1, the participants completed a drawing task, followed by the placement task. However, the data from the drawing task were not analyzed, since the focus was primarily on the results of the placement task. The only other difference between the present experiment and Experiment 1 was that the order of the stimulus sets was counterbalanced across participants.

Coding and measurement. The displays were photographed and then reproduced as PowerPoint slides for coding. As in Experiment 1 , the displays were coded by two independent raters to evaluate search asymmetries and for the use of eccentricity and orientational heterogeneity. In addition, we included objective measures of

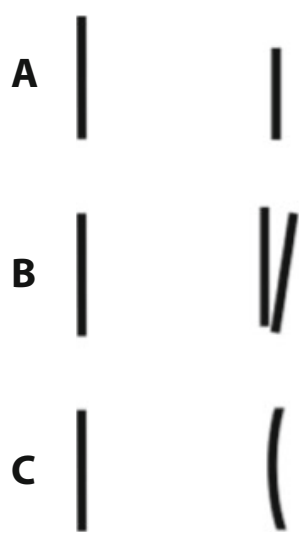

Figure 3. Stimulus sets used for the placement task in Experiment 3. (A) Long/short. (B) Double/single. (C) Curved/straight. 
A

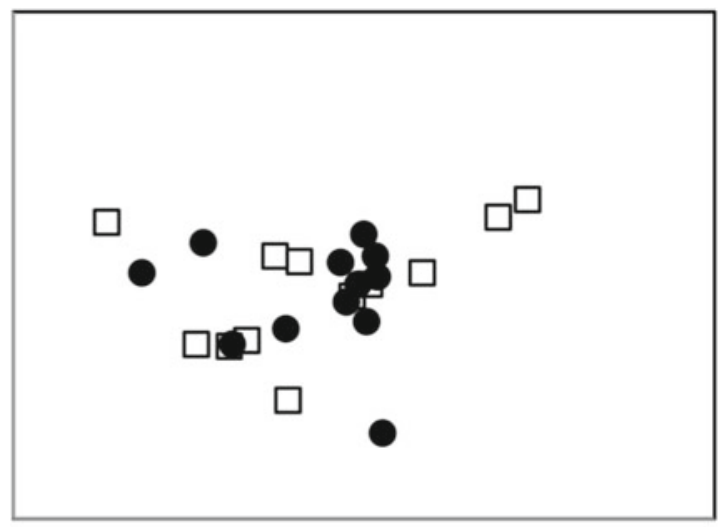

B

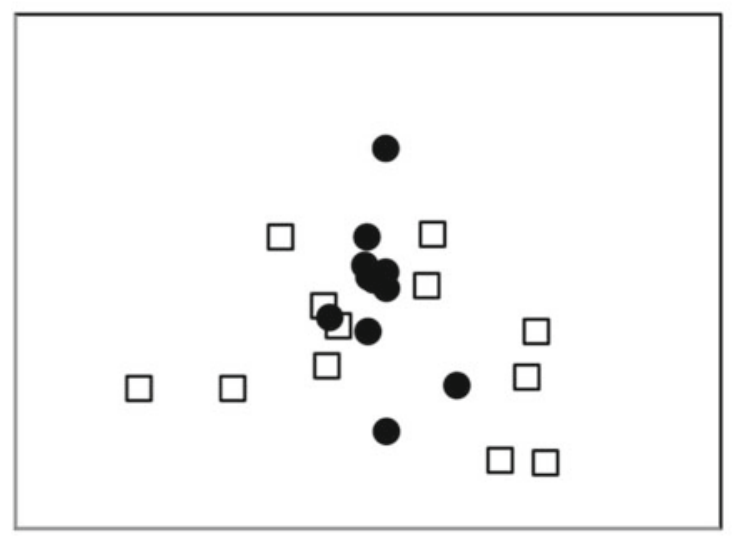

C

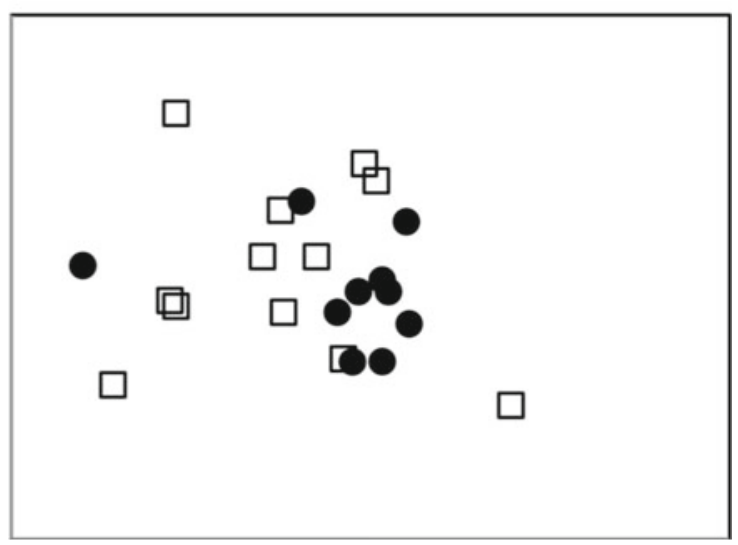

Figure 4. Target positions for easy (black circles) and hard (white squares) search displays associated with the long/short (A), single/double (B), and curved/straight (C) stimulus sets.

both eccentricity and orientational heterogeneity. Specifically, we used absolute distance of the target from screen center to measure target eccentricity and the average between-item difference in rotation among items as a measure of orientational heterogeneity.

\section{Results}

Overall, the results of Experiment 3 were consistent with the findings of Experiment 1. As an overview, the participants intuited that search difficulty would be influenced by the presence or absence of a feature in the target (i.e., search asymmetries). This was the case for two of the three stimulus sets we tested. In addition, we again found that the participants intuited that search difficulty is influenced by target eccentricity, organization, and orientation. These findings will be discussed in more detail below.

Presence versus absence of a feature. Table 6 shows the frequencies with which the participants used each stimulus in a given stimulus pair as a target. The participants were assigned a score of " 1 " if they chose the target with a feature present and a " 0 " if they chose the target with the feature absent. The scores were then submitted to an ANOVA with difficulty (easy vs. hard) and stimulus set (long/short lines vs. double/single lines vs. curved/straight lines) as within-participants factors. Inspection of Table 6 reveals that the participants used the item with an extra feature present as the target on a larger proportion of the easy displays (.61) than of the hard displays $(.25)[F(1,11)=$ $\left.5.357, M S_{\mathrm{e}}=0.438, p<.05\right]$. The main effect of stimulus set and the interaction did not reach significance $\left(F_{\mathrm{S}}<\right.$ $2.557, p \mathrm{~s}>.10)$. The effect of difficulty was evident with the long/short and double/single stimulus sets, but not with the curved/straight stimulus set (see Table 6). Thus, overall, the data show that the participants used standard search asymmetries to influence search difficulty, although this did not apply to one of the stimulus sets.

Eccentricity. We first assessed target eccentricity using raters, as we did in Experiment 1. The interrater agreement was nearly perfect $(r=.99)$, and so the scores were averaged across raters. The scores were then submitted to an ANOVA with difficulty (easy vs. hard) and stimulus set (long/short lines vs. double/single lines vs. curved/straight lines) as within-participants factors. Table 7 shows the mean scores for each condition. Inspection of the table reveals that the targets were placed farther away from center in the hard search displays than in the easy search displays $\left[F(1,11)=23.8, M S_{\mathrm{e}}=2.20, p<.01\right]$. There was no main effect of stimulus set $(F<1)$ and a nonsignificant stimulus set $\times$ difficulty interaction $[F(2,22)=$ 2.3, $\left.M S_{\mathrm{e}}=2.03, p=.13\right]$. The marginal interaction can be attributed to the fact that the difference in eccentricity scores between easy and hard conditions emerged statisti-

Table 6

Frequencies With Which Targets in Easy and Hard Search Displays Were Defined by the Presence or Absence of a Feature for Each of the Stimulus Sets Used in Experiment 3

\begin{tabular}{ccc} 
for Each of the Stimulus Sets Used in Experiment 3 \\
\cline { 2 - 3 } Stimulus Set & \multicolumn{2}{c}{ Difficulty } \\
\cline { 2 - 3 } Easy & Hard \\
\hline Fong/short & 8 & 2 \\
Feature present & 4 & 10 \\
Feature absent & & \\
Double/single & 9 & 2 \\
Feature present & 3 & 10 \\
Feature absent & & \\
Curved/straight & & 5 \\
Feature present & 5 & 7 \\
Feature absent & 7 & \\
\hline
\end{tabular}


Table 7

Mean Scores on the Eccentricity and Orientational Heterogeneity Dimensions As a Function of Stimulus Set and Search Difficulty in Experiment 3

\begin{tabular}{lrrrrrr}
\hline & \multicolumn{2}{c}{ Difficulty } & & & \\
\cline { 2 - 3 } \multicolumn{1}{c}{ Dimension } & Easy & Hard & Difference & $t$ & $p$ \\
\hline Eccentricity & & & & & \\
$\quad$ Long/short & 2.5 & 3.3 & 0.7 & 1.3 & .22 \\
Double/single & 1.8 & 3.8 & 2.0 & 3.7 & .00 \\
$\quad$ Curved/straight & 1.7 & 4.1 & 2.4 & 3.6 & .00 \\
Orientational heterogeneity & & & & \\
$\quad$ Long/short & 2.5 & 4.8 & 2.3 & 3.0 & .01 \\
Double/single & 2.3 & 5.8 & 3.5 & 6.5 & .00 \\
Curved/straight & 2.8 & 4.8 & 2.0 & 2.6 & .03 \\
\hline
\end{tabular}

Note-Difference scores are calculated as scores in the hard condition minus the scores in the easy condition. All $t$ tests are repeated measures, two-tailed tests.

cally only in the double/single and curved/straight stimulus sets and not in the long/short set (see Table 7).

Having replicated the effect of difficulty on target eccentricity using subjective ratings of eccentricity as in Experiment 1 , we next assessed eccentricity more objectively by measuring the distance of the target from the center of each display. The location of each target in the easy and hard conditions for each stimulus set is shown in Figure 4. As can be seen in the figure, the targets associated with easy displays were located more centrally than were the targets associated with the hard displays.

In order to report a meaningful measure of a target's distance from center, we measured the target's horizontal and vertical distances from center and then converted these values to proportions of the maximum horizontal and vertical displacement from center. We then used the Pythagorean theorem to compute the final proportional distance of the target from center as the measure of eccentricity. This resulted in a measure of eccentricity that was independent of the specific size of our display. The proportional distances from center of targets in hard and easy displays for each stimulus set are shown in Figure 5. The scores were submitted to an ANOVA with difficulty (easy vs. hard) and stimulus set (long/short lines vs. double/single lines vs. curved/straight lines) as withinparticipants factors. The analysis confirmed that, overall, there was a substantial effect of difficulty $[F(1,11)=$ $\left.25.0, M S_{\mathrm{e}}=0.033, p<.001\right]$, whereby the targets in the easy displays were closer to the center than were the targets in the hard displays. There was no difference in target eccentricities across stimulus sets and no significant interaction $(F \mathrm{~s}<1.2, p \mathrm{~s}>.32)$. Thus, the results of the objective measure were completely consistent with the results of the subjective assessment provided by the raters. Also consistent with the subjective ratings was that substantial differences in eccentricity between easy and hard displays were found for the double/single and curved/straight stimulus sets $(p$ s $<.01)$, but not for the long/short stimulus set ( $p=.11)$. Overall, however, the results clearly show that, as in Experiment 1, the participants used target eccentricity to influence search difficulty.
Orientational heterogeneity. We again began with the subjective ratings of orientational heterogeneity. The scores of the two raters once more showed a high level of agreement $(r=.86)$, and so the scores were averaged across raters, and the mean scores for each condition are shown in Table 7 . The data were submitted to a 2 (difficulty: easy vs. hard) $\times 3$ (stimulus set: long/short lines vs. double/single lines vs. curved/straight lines) repeated measures ANOVA. The analysis revealed a main effect of difficulty $\left[F(1,11)=39.8, M S_{\mathrm{e}}=3.02, p<.001\right]$, with there being more orientational heterogeneity in the hard displays than in the easy displays. Neither the main effect of stimulus set nor the difficulty $\times$ stimulus set interaction reached significance $\left(F_{\mathrm{s}}<1.21, p \mathrm{~s}>.31\right)$. Table 7 also shows significant differences in scores between the easy and hard displays for each stimulus set.

As an objective measure of orientational heterogeneity, we recorded the angle of rotation of each of the objects in a given display. We then computed the difference in orientation between each pair of objects by computing the magnitude (in degrees) of the acute angle between the orientations of each pair of objects. All of these pairwise differences were then averaged to arrive at the mean orientational difference between items, which served as our measure of orientational heterogeneity. The scores were submitted to an ANOVA with difficulty (easy vs. hard) and stimulus set (long/short lines vs. double/single lines vs. curved/straight lines) as within-participants factors. The mean orientational difference between items, averaged across participants, for each condition is shown in the top panel of Figure 6. As can be seen in the figure, the mean orientational difference between items was greater in hard displays than in easy displays $\left[F(1,11)=24.5, M S_{\mathrm{e}}=\right.$ $1,054, p<.001]$. The mean orientational difference between items also differed across stimulus sets $[F(2,22)=$

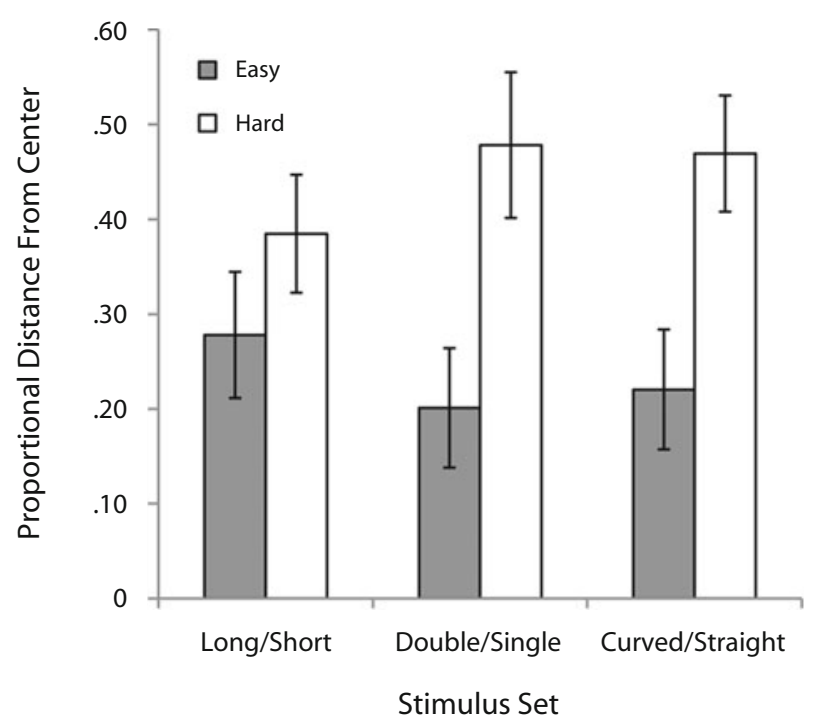

Figure 5. Mean proportional distance from the center of the target for easy and hard search displays associated with each of the stimulus sets. Error bars reflect one standard error of the mean. 

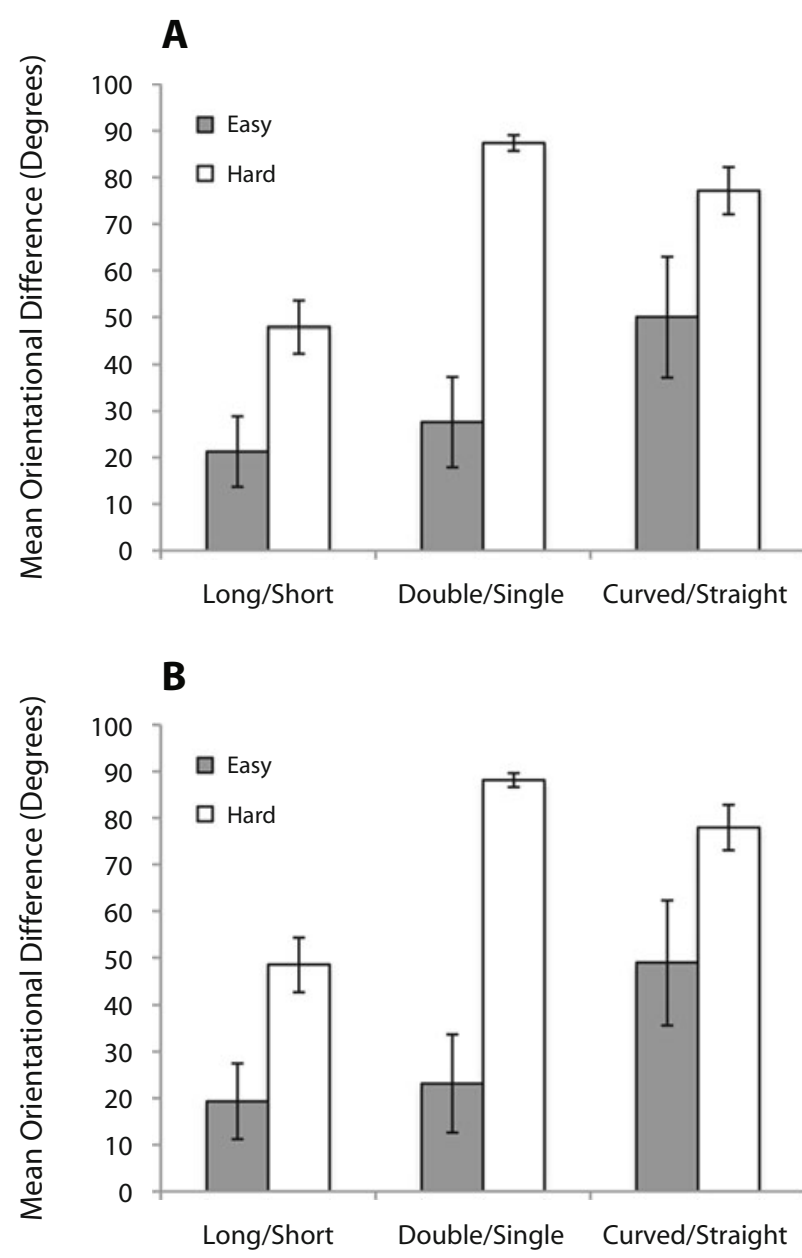

Figure 6. Mean orientational differences (degrees) for easy and hard search displays associated with each of the stimulus sets. (A) Mean orientational differences between all items in the displays. (B) Mean orientational differences between the distractor items in the displays only. Error bars reflect one standard error of the mean.

$\left.19.9, M S_{\mathrm{e}}=281.4, p<.001\right]$, with the least orientational heterogeneity in the long/short stimulus set. There was a marginally nonsignificant interaction between difficulty and stimulus set $\left[F(2,22)=2.6, M S_{\mathrm{e}}=837.9, p=.098\right]$, indicating slightly larger effects of difficulty in the long/ short and double/single stimulus sets $(p \mathrm{~s}<.01)$ than in the curved/straight stimulus set $(p=.064)$ as indexed by one-tailed paired-sample $t$ tests. Overall, therefore, the results with the objective and subjective measures were consistent with each other and with the results of Experiment 1 , indicating that the participants used orientational heterogeneity to influence the difficulty of search.

Having objectively measured the orientation of each item on a given display, it was possible to exclude the target item and assess the mean orientational difference between only the distractor items as an index of distractordistractor similarity (see Duncan \& Humphreys, 1989). These scores are shown in the bottom panel of Figure 6. Not surprisingly, since most of the items in the displays were distractors, the results were very similar to those obtained when all items were considered in our assessment of orientational heterogeneity (in the top panel). Thus, the present findings clearly show that the participants effectively used distractor-distractor similarity when varying search difficulty, dovetailing with recent empirical findings (e.g., van Zoest, Giesbrecht, Enns, \& Kingstone, 2006; see also Kingstone \& Bischof, 1999).

Combining target eccentricity and orientational heterogeneity. Since the participants used both target eccentricity and orientational heterogeneity to vary search difficulty, it seems reasonable to plot these two factors on a single scatterplot. A set of scatterplots of the two factors for each stimulus set is shown in Figure 7. As can be seen in the figure, the easy (black circles) and hard (white squares) search displays formed two distinct groups, with the easy search displays being localized mostly on the bottom left of the plots and the hard displays being localized mostly on the top right of the plots.

\section{EXPERIMENT 4}

Taken together, Experiments 1 and 3 showed that participants use search asymmetries (the presence vs. absence of a feature) when varying the difficulty of search. One exception was that the participants in Experiment 3 did not use the search asymmetries with the curved/straight stimulus set. This may have been because the participants simply opted to use other factors such as target eccentricity and orientation, to vary search difficulty. On the other hand, it may show limits to people's understanding and use of search asymmetries. It also remains unclear whether people have explicit knowledge of the influence of asymmetries on search difficulty or whether they use the factor in a more implicit manner as they physically create the display. These issues were addressed in Experiment 4.

To address these issues, we introduced a forced choice hiding paradigm. In the forced choice hiding paradigm, the participants were shown each pair of asymmetry stimuli used in Experiments 1 and 3 and were asked which item in each pair they would use as a target and which item they would use as a distractor to make the search either easy or hard. Critically, the participants were not given an opportunity to create the search displays, and so their answers would reflect their explicit knowledge about how search asymmetries influence search difficulty. In addition, by not allowing the participants to use any other factor to vary search difficulty, we could evaluate whether they would demonstrate knowledge of the role of asymmetries in the curved/straight stimulus set, which they did not demonstrate using in Experiment 3.

\section{Method}

Participants. Thirty-six undergraduate students at the University of Waterloo participated in the study in a 20-min session in exchange for a partial course credit. Eighteen participants were required to make the search easy, and 18 were required to make the search hard.

Materials. The asymmetry stimulus sets from Experiment 1 (circle/circle with a line) and Experiment 3 (long/short, double/ single, curved/straight) were used in Experiment 4. 


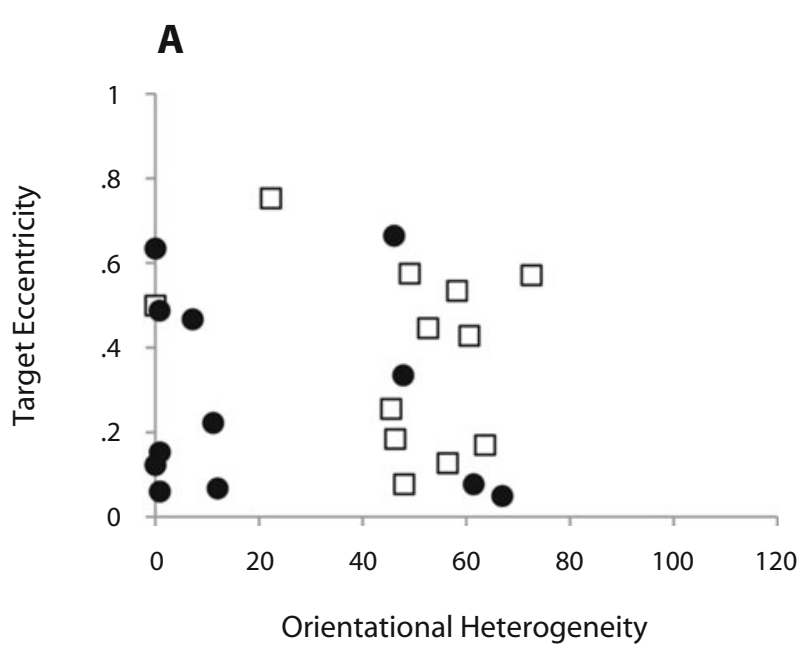

B
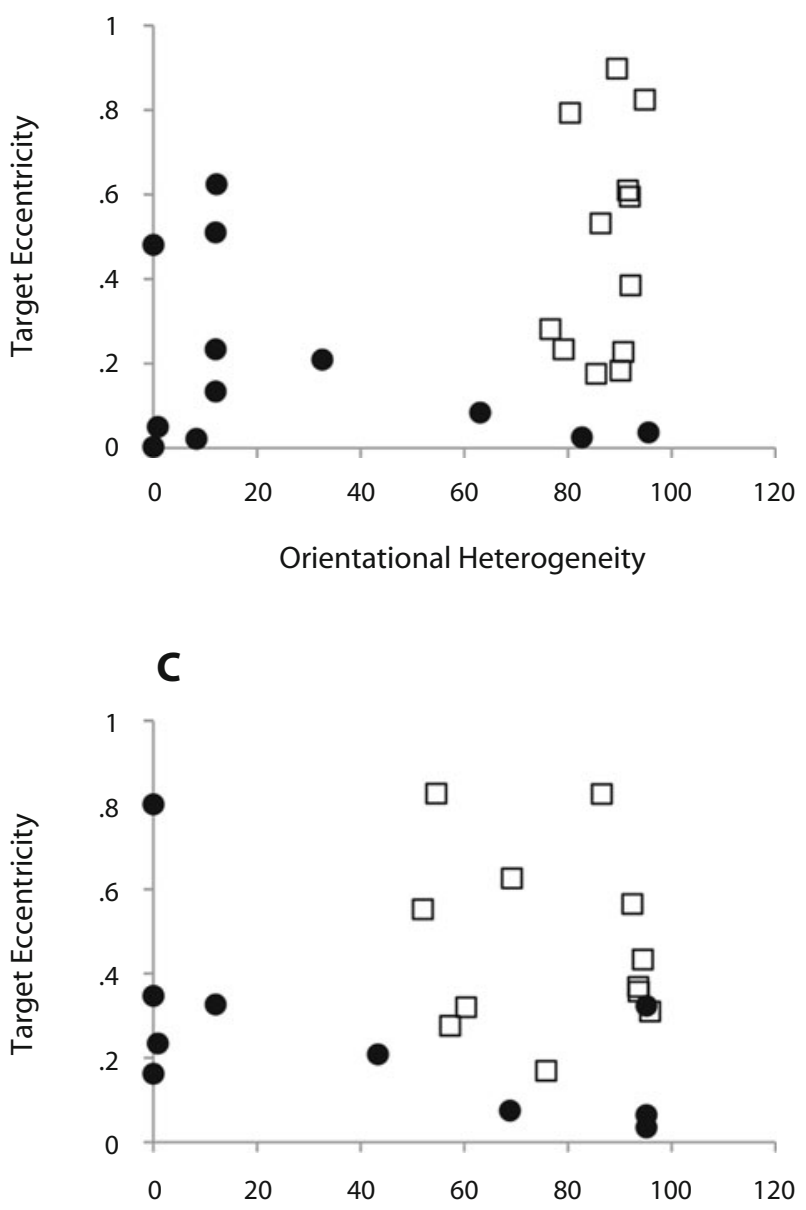

Orientational Heterogeneity

Figure 7. Target eccentricity (proportional distance from center) and orientational heterogeneity (average orientational difference between items in degrees) for easy (black circles) and hard (white squares) search displays associated with the long/short (A), single/double (B), and curved/straight (C) stimulus sets.
Procedure. Upon entering the lab, the participants were randomly assigned to either the group asked to make easy searches or the group asked to make hard searches. To help the participants understand the task, they were first shown an example of a standard search display containing a unique target item (an "X") among a group of distractor items (a group of "O"s). The participants were then shown a pair of shapes from a given asymmetry set and were required to choose which shape in the pair they would use as the target in a hypothetical visual search display such that it would be either easy (easy group) or hard (hard group) to find. The order in which the stimulus sets were presented was random, as was the left/ right arrangement of the paired shapes.

\section{Results and Discussion}

The frequencies with which each of the stimuli in the four asymmetry pairs was chosen to be a target for easy and hard search displays are shown in Table 8 . As can be seen in the table, the participants clearly intuited that presence versus absence of a feature influences search difficulty. The results show that for each of the stimulus sets, the participants were more likely to choose the stimulus with the additional feature as a target to make search easy and the stimulus with the absence of a feature as the target to make search hard. Since, in this experiment, search difficulty was varied between participants, it was possible to use the chi squared statistic to analyze the choices for each stimulus set. The analysis revealed significant effects of search difficulty for each of the stimulus sets [circle/circle-line, $\chi^{2}(1)=28.235, p<.001$; long/short, $\chi^{2}(1)=16.762, p<.001$; double/single, $\chi^{2}(1)=15.184$, $p<.001 ;$ curved/straight, $\chi^{2}(1)=4.571, p=.033$.

Importantly, the results of Experiment 4 clearly showed that the participants were even able to intuit that a curved line among straight lines would be easier to find than a straight line among curved lines. We find this interesting because the curved/straight asymmetry seems much more subtle than the other asymmetries we tested, yet the participants were still able to intuit this asymmetry. Recall that when the participants created displays using this asymmetry in Experiment 3, they did not use the asymmetry to vary search difficulty. One interpretation of those results was that there were limits to the participants' knowledge of the role of asymmetries in search and they did not have intuitive knowledge about more subtle asymmetries. The results of Experiment 4 clearly show, however, that this was not the case. Instead, the results support the alternative possibility that the participants had knowledge of even subtle asymmetries but that they might not have opted to use them to vary search difficulty when alternative factors (e.g., eccentricity and orientation) were more salient.

\section{GENERAL DISCUSSION}

To evaluate whether participants use factors known to govern search difficulty when they are asked to conceal objects to various degrees, we had the participants in Experiment 1 create search displays in which the targets were either easy or hard to find. Analyses of the easy and hard search displays revealed that the participants reliably used a number of factors known to influence search difficulty, 
Table 8

Frequencies With Which Targets in Easy and Hard Search Displays Were Defined by the Presence or Absence of a Feature for Each of the Stimulus Sets Used in Experiment 4

\begin{tabular}{crr}
\hline & \multicolumn{2}{c}{ Difficulty } \\
\cline { 2 - 3 } Stimulus Set & Easy & Hard \\
\hline Circle/circle with line & & \\
Feature present & 15 & 16 \\
Feature absent & 1 & \\
Long/short & & 0 \\
Feature present & 11 & 16 \\
Feature absent & 5 & \\
Double/single & & 2 \\
Feature present & 13 & 14 \\
Feature absent & 3 & \\
Curved/straight & & 4 \\
Feature present & 10 & 12 \\
Feature absent & 6 & \\
\hline
\end{tabular}

such as (1) the eccentricity of the target, (2) the orientation of the search items (e.g., distractor-distractor similarity), (3) the visual similarity between the target and the distractors, and (4) the presence or absence of a feature in the target object relative to the distractor objects. In addition, the participants showed a trend toward using emotional valence to vary search difficulty, although differently than it has typically been shown to influence search. Finally, we found that the participants did not consistently use alphanumeric category to vary search difficulty. As a confirmation that the participants in Experiment 1 were successful at making easy and hard search displays, we had another group of participants in Experiment 2 search the displays created by the participants in Experiment 1. The results clearly showed that search was more difficult in the displays created to be hard than in those created to be easy searches by the participants in Experiment 1. Experiments 3 and 4 demonstrated further that participants have knowledge about the role of the presence versus absence of a feature. This includes some knowledge of even the relatively subtle search asymmetry between curved and straight lines, although the extent to which people intuited or used this subtle asymmetry has varied across studies. Taken together, the results suggest that people have considerable knowledge about the factors that govern search difficulty-even about subtle factors often varied in laboratory search studies - and that these factors are used when individuals make decisions about how to conceal objects.

\section{A Search-Based Theory of Visual Concealment}

The present findings indicate that visual search theory can be extended to form a foundation for a theory of visual concealment. We propose a preliminary theory of concealment based on the fundamental assumption that participants have access to the factors that influence basic mechanisms of visual search. We suggest that participants can access these factors in three different ways. First, participants can access propositional knowledge (see Pylyshyn, 1973, 1981) about some factors that influence search. This explicit knowledge is likely abstracted from prior experience with hiding and finding things in the real world. For instance, most people know from prior experience that placing objects in more peripheral areas of the environment will make them more difficult to find than placing them centrally. Second, participants can use visual images (see Kosslyn, 1976, 1980) to create mental simulations of hiding scenarios and internally test how effectively their basic search mechanisms will guide them to the target. Third, participants can also manipulate the external environment and try different scenarios to test how effectively their basic search mechanisms will guide them to the target.

The results of Experiments 1 and 3 could be explained by any of these three ways in which participants might access the factors that govern search. The results of Experiment 4, however, revealed that manipulating the external environment to test different search scenarios is not necessary, since the participants demonstrated clear knowledge of search asymmetries without physically creating displays. We should also note that informal observation of the participants' behavior in Experiments 1 and 3 suggests that manipulating the external environment is relatively less important than propositional knowledge and mental imagery, since the participants did not typically try different configurations of items. Instead, the participants almost exclusively just placed the items the first time to create a given configuration, which suggests that they might have been working from a mental image of the displays.

There are likely limits to the accessibility of factors that govern concealment and search. Propositional knowledge of a given factor either might not be available to an individual or, although available, might not be accessible in a certain situation (see Thomson \& Tulving, 1970; Tulving $\&$ Pearlstone, 1966). In addition, it might be difficult for an individual to accurately simulate and test (through imagery or physical manipulation) how effectively his/her basic mechanisms of search will guide attention, because simulating a search scenario entails knowing where the target is and this likely biases one's evaluation of search difficulty. It would be interesting to explore these limitations in future research.

Finally, our theory of concealment distinguishes between the factors that people have potential access to and those that they actually use in a given situation. Our experiments suggest that participants might not use all of the factors that they have access to and that they might, instead, just focus on the salient factors. For instance, the participants in Experiment 3 did not use search asymmetries when they created displays with the curved/straight stimulus set, although the participants in Experiment 4 showed clear knowledge of the impact of that particular asymmetry on search. In addition, inspection of Table 2 shows that the participants used eccentricity, organization, and orientation less with the alphanumeric stimulus set than with the asymmetry-shape and emotional-valence sets, very possibly because the alphanumeric stimulus set allowed the participants to vary target-distractor similarity, which is arguably a very salient factor. Finally, Experiment 3 revealed that target eccentricity and orientational heterogeneity are not correlated and, thus, are likely varied independently, with some participants primarily varying 
orientational heterogeneity and others primarily varying eccentricity.

It is worth highlighting that central to our theory of concealment is the idea that visual concealment behavior is indirectly scaffolded on the basic mechanisms of visual search. As such, we refer to this initial theory as the search-based theory of visual concealment. We hasten to add that our investigation provides only the initial steps toward a complete theory of concealment, which will necessarily include a critical role for occlusion and a description of how concealment occurs in more real-world situations. Nonetheless, our hope is that the present findings open the door for future research on this topic.

\section{Future Directions}

The present findings suggest several interesting avenues for future investigation. First, given that people will not always use all of the factors that they know influence concealment, it would be useful to know how people choose which factors to use in a given concealment situation. A second issue for future investigation concerns how explicit people's knowledge of various search factors actually is. It could be that some factors are known more explicitly, whereas others are known more implicitly. A third future direction would be to investigate people's intuitions about how various factors influence search efficiency, rather than just search difficulty, which we investigated in the present studies. The visual search literature distinguishes between search difficulty, which is typically measured by overall RTs, and search efficiency, which is measured by the slopes of the function relating search times to the number of items in the displays. Although we believe that the differences we found between the easy and hard displays reflect knowledge about the factors that influence search efficiency, and not just search difficulty, we did not directly manipulate the number of items in the displays, and so we cannot draw strong links between people's intuitions and search efficiency. In future studies, it would be useful to vary the number of items in the displays to directly investigate people's intuitions about factors that influence search efficiency. A fourth future direction would be to investigate other factors known to govern search difficulty/efficiency, such as conjunction versus feature search (Treisman \& Gelade, 1980) and very subtle factors such as linear separability in color space (Bauer, Jolicœur, \& Cowen, 1998). Finally, it would be worthwhile to evaluate which factors people use to conceal objects in real-world settings, such as hiding objects in travel luggage. Such real-world situations might reveal that people typically use occlusion and consider more high-level factors, such as people's expectations (schemata) about where objects are typically placed (or not placed), as well as expectations about where a potential searcher might look. In light of the success of our initial studies, the present concealment paradigm may be a useful tool for investigating these new directions.

\section{AUTHOR NOTE}

This research was supported by grants to D.S. and A.K. from the Natural Sciences and Engineering Research Council. Correspondence should be sent to D. Smilek, Department of Psychology, University of Waterloo, 200 University Avenue West, Waterloo, ON, N2L 3G1 Canada (e-mail: dsmilek@uwaterloo.ca).

\section{REFERENCES}

Bauer, B., Joliceur, P., \& Cowen, W. B. (1998). The linear separability effect in color visual search: Ruling out the additive color hypothesis. Perception \& Psychophysics, 60, 1083-1093.

BRAND, J. (1971). Classification without identification in visual search. Quarterly Journal of Experimental Psychology, 23, 178-186.

Bundesen, C. (1990). A theory of visual attention. Psychological Review, 97, 523-547.

Carrasco, M., Evert, D. L., Chang, I., \& Katz, S. M. (1995). The eccentricity effect: Target eccentricity affects performance on conjunction searches. Perception \& Psychophysics, 57, 1241-1261.

Carrasco, M., \& Frieder, K. S. (1997). Cortical magnification neutralizes the eccentricity effect in visual search. Vision Research, 37, 63-82.

Carrasco, M., McLean, T. L., Katz, S. M., \& Frieder, K. S. (1998). Feature asymmetries in visual search: Effects of display duration, target eccentricity, orientation and spatial frequency. Vision Research, 38, 347-374

Carrasco, M., Talgar, C. P., \& Cameron, E. L. (2001). Characterizing visual performance fields: Effects of transient covert attention, spatial frequency, eccentricity, task and set size. Spatial Vision, 15, 61-75.

Carrasco, M., \& Yeshurun, Y. (1998). The contribution of covert attention to the set-size and eccentricity effects in visual search. Journal of Experimental Psychology: Human Perception \& Performance, 24, 673-692.

Coltheart, M., Rastle, K., Perry, C., Langdon, R., \& Ziegler, J. (2001). DRC: A dual route cascaded model of visual word recognition and reading aloud. Psychological Review, 108, 204-256.

Duncan, J. (1983). Category effects in visual search: A failure to replicate the "oh-zero" phenomenon. Perception \& Psychophysics, 34, 221-232.

Duncan, J., \& Humphreys, G. (1989). Visual search and stimulus similarity. Psychological Review, 96, 433-458.

Eastwood, J. D., Smilek, D., \& Merikle, P. M. (2001). Differential attention guidance by unattended faces expressing positive and negative emotion. Perception \& Psychophysics, 63, 1004-1013.

Fox, E., Lester, V., Russo, R., Bowles, R. J., Pichler, A., \& DutTON, K. (2000). Facial expressions of emotion: Are angry faces detected more efficiently? Cognition \& Emotion, 14, 61-92.

Frischen, A., Eastwood, J. D., \& Smilek, D. (2008). Visual search for faces with emotional expressions. Psychological Bulletin, 134, 662-676.

Gerritsen, C., Frischen, A., Blake, A., Smilek, D., \& Eastwood, J. D. (2008). Visual search is not blind to emotion. Perception \& Psychophysics, 70, 1047-1059.

Gibson, E. J., Osser, H., Schiff, W., \& Smith, J. (1963). An analysis of critical features of letters, tested by a confusion matrix. In $A$ basic research program on reading (Cooperative Research Project No. 639). Washington, DC: U.S. Office of Education.

Gleitman, H., \& Jonides, J. (1978). The effect of set on categorization in visual search. Perception \& Psychophysics, 24, 361-368.

Hansen, C. H., \& Hansen, R. D. (1988). Finding the face in the crowd: An anger superiority effect. Attitudes \& Social Cognition, 54, 917924.

Hooge, I. T. C., \& ERKelens, C. J. (1998). Adjustment of fixation duration in visual search. Vision Research, 39, 1567-1575.

Hunt, A. R., Cooper, R. M., Hungr, C., \& Kingstone, A. (2007). The effect of emotional faces on eye movements and attention. Visual Cognition, 15, 513-531.

INGLING, N. W. (1972). Categorization: A mechanism for rapid information processing. Journal of Experimental Psychology, 94, 239-243.

Jonides, J., \& Gleitman, H. (1972). A conceptual category effect in visual search: $\mathrm{O}$ as letter or as digit. Perception \& Psychophysics, 12, 457-460.

Jonides, J., \& Gleitman, H. (1976). The benefit of categorization in visual search: Target location without identification. Perception \& Psychophysics, 20, 289-298. 
Kingstone, A., \& Bischof, W. F. (1999). Perceptual grouping and motion coherence in visual search. Psychological Science, 10, 151156.

Kosslyn, S. M. (1976). Can imagery be distinguished from other forms of internal representation? Evidence from studies of information retrieval times. Memory \& Cognition, 4, 291-297.

Kosslyn, S. M. (1980). Image and mind. Cambridge, MA: Harvard University Press.

Moraglia, G. (1989). Display organization and the detection of horizontal line segments. Perception \& Psychophysics, 45, 265-272.

Ohman, A., Lundqvist, D., \& Esteves, F. (2001). The face in the crowd revisited: A threat advantage with schematic stimuli. Journal of Personality \& Social Psychology, 80, 381-396.

Olds, E. S., Cowan, W. B., \& Jolicceur, P. (1999). Spatial organization of distractors in visual search. Canadian Journal of Experimental Psychology, 53, 150-159.

Purcell, D. G., Stewart, A. L., \& Skov, R. B. (1996). It takes a confounded face to pop out of a crowd. Perception, 25, 1091-1108.

Pylyshyn, Z. W. (1973). What the mind's eye tells the mind's brain: A critique of mental imagery. Psychological Bulletin, 80, 1-24.

Pylyshyn, Z. W. (1981). The imagery debate: Analogue media versus tacit knowledge. Psychological Review, 88, 16-45.

Rumelhart, D. E., \& McClelland, J. L. (1982). An interactive activation model of context effects in letter perception: II. The contextual enhancement effect and some tests and extensions of the model. Psychological Review, 89, 60-94.

Rumelhart, D. E., \& SiPle, P. (1974). The process of recognizing tachistoscopically presented words. Psychological Review, 81, 99-118.

Schneider, W., Eschman, A., \& Zuccolotto, A. (2001). E-Prime user's guide. Pittsburgh: Psychology Software Tools Inc.

Smilek, D., Dixon, M. J., \& Merikle, P. M. (2006). Revisiting the category effect: The influence of meaning and search strategy on the efficiency of visual search. Brain Research, 1080, 73-90.

Thomson, D. M., \& Tulving, E. (1970). Associative encoding and retrieval of weak and strong cues. Journal of Experimental Psychology, 86, 255-262.

Treisman, A. M., \& Gelade, G. (1980). A feature-integration theory of attention. Cognitive Psychology, 12, 97-136.

Treisman, A. [M.], \& Gormican, S. (1988). Feature analysis in early vision: Evidence from search asymmetries. Psychological Review, 95 , $15-48$.

Treisman, A. [M.], \& Souther, J. (1985). Search asymmetry: A diagnostic for preattentive processing of separable features. Journal of Experimental Psychology: General, 114, 285-310.

Tulving, E., \& PeArlstone, Z. (1966). Availability versus accessibility of information in memory for words. Journal of Verbal Learning \& Verbal Behavior, 5, 381-391.

van Zoest, W., Giesbrecht, B., Enns, J. T., \& Kingstone, A. (2006). New reflections on visual search: Inter-item symmetry matters! Psychological Science, 17, 535-542.

Wolfe, J. M. (1994). Guided Search 2.0: A revised model of visual search. Psychonomic Bulletin \& Review, 1, 202-238.

Wolfe, J. M. (1998). Visual search. In H. Pashler (Ed.), Attention (pp. 13-73). London: University College London Press.

Wolfe, J. M., CAVE, K. R., \& Franzel, S. L. (1989). Guided search: An alternative to the feature integration model for visual search. Journal of Experimental Psychology: Human Perception \& Performance, 15, 419-433.

Wolfe, J. M., Friedman-Hill, S. R., Stewart, M. I., \& O’Connell, K. M. (1992). The role of categorization in visual search for orientation. Journal of Experimental Psychology: Human Perception \& Performance, 18, 34-49.

\section{NOTES}

1. The Rumelhart and Siple (1974) stimulus set consists only of alphabetic characters; therefore, we extended the feature set to numbers $0-9$. For the present purposes, we ignored increases in feature similarity that arise from participants rotating the characters (e.g., $\mathrm{W}-\mathrm{M}, \mathrm{N}-\mathrm{Z}$ ).

2. In the alphanumeric set, there were 17 instances where displays were created using characters that differed in terms of up to two features, using the Rumelhart and Siple (1974) feature set (i.e., O/Q, F/E, and $\mathrm{I} / 1)$. All of these displays were in the hard condition, and in 12 of the 17 displays, the character with the fewer number of features was the target $\left(\chi^{2}=20, p<.001\right)$.

(Manuscript received December 5, 2008; revision accepted for publication June 16, 2009. 